\title{
An Evaluation of the Use of Calcium, Potassium and Silicon for the Management of Diaphorina citri Populations in Tahiti Lime Trees
}

\author{
Augusto RAMÍREZ-GODOY, Ginna PUENTES-PÉREZ, \\ Hermann RESTREPO-DÍAZ*
}

\author{
Universidad Nacional de Colombia, Facultad de Ciencias Agrarias, Departamento de Agronomía, Carrera 30 No 45-03 Edificio 500, Bogotá, \\ Colombia; augramirezg@unal.edu.co;gmpuentesp@unal.edu.co; hrestrepod@unal.edu.co ('corresponding author)
}

\begin{abstract}
The recent appearance of the disease known as huanglongbing (HLB) in Colombia has caused the demand for alternative control methods for Diaphorina citri (i.e., the disease vector). Specifically, the use of nutrients, such as calcium (Ca), potassium $(\mathrm{K})$ and silicon $(\mathrm{Si})$, may provide some degree of plant defense against herbivory. One set of experiments (in the form of two separate experiments) was conducted on two different farms in the municipality of Jerusalén to study the effects of foliar and soil applications of nutrients $(\mathrm{Ca}, \mathrm{K}$ and $\mathrm{Si}$ ) on controlling the population dynamics of Diaphorina citri. Tahiti lime trees were treated as follows: i) untreated trees (absolute control); ii) clothianidin at a dose of $50 \mathrm{~g}$ active ingredient per hectare (chemical control); iii) Ca, K and Si foliar applications (at doses of $3 \mathrm{~mL}, 3 \mathrm{~g}$ and $2 \mathrm{~mL}$ of the commercial compound used per liter of $\mathrm{H} 2 \mathrm{O}$, respectively); and iv) soil application of potassium nitrate and potassium silicate (1 kg of commercial fertilizer per tree). Foliar sprays were carried out at 0 and 4 weeks after treatment (WAT) began; meanwhile, soil fertilization occurred at the beginning of the trial (i.e., 0 WAT). The results showed that differences were observed only in adults at 7 WAT, and the foliar calcium and silicon applications resulted in the lowest number of individuals (i.e., 1.13 per flush) compared with untreated trees (i.e., 3.13 per flush). The foliar clothianidin, Ca, Si and K sprays also affected the total number of nymphs. Additionally, Tahiti lime trees treated with either silicon or insecticide had fewer eggs than did trees in the other treatments. The use of these mineral nutrients showed a similar efficacy when compared to clothianidin, indicating that these mineral nutrients can enhance plant resistance. These observations suggest that foliar applications of $\mathrm{K}, \mathrm{Ca}$ and $\mathrm{Si}$ could be considered as complementary tools within an integrated management program for D. citri in Colombia.
\end{abstract}

Keywords: Asian citrus psyllid; foliar fertilization; plant resistance; percentage of efficacy; mineral nutrients

\section{Introduction}

A severe decline has recently taken place in the citrus industry of many countries as a result of huanglongbing disease (HLB) (Gottwald, 2010), which is caused by phloem-limited bacteria (Jagoueix et al., 1994). There is currently no cure for this disease, thus management is mainly focused on the management of its vector, the Asian citrus psyllid (ACP), Diaphorina citri Kuwayama (Hemiptera: Psyllidae) (Canales et al., 2016). The life cycle of $D$. citri is closely related to the presence of new growth flushes, which are necessary for oviposition and nymph development. Consequently, D. citri populations decrease when flushing rates are low or nonexistent (Pluke et al. 2008). In the tropics, citrus flush development is conditioned by regional precipitation patterns throughout the year (Orduz-Rodríguez et al., 2010; Orduz-Rodríguez and Garzón, 2012).

In the tropics, the continuous production of new flushes allows $D$. citri populations to remain relatively high at various times of the year (Pluke et al., 2008), causing that chemical control would be difficult because new growth is not protected by insecticides (Tsagkarakis et al., 2012). Additionally, the frequent use of insecticides generates environmental concerns, such as negative effects on beneficial arthropods or increased resistance of $D$. citri to insecticides. In this aspect, the development of alternative forms of management that favor host resistance to the insect pest significantly contributes to the improvement of integrated management programs for D. citri (Hall et al., 2013).

The application of nutrients is a method that helps mitigate biotic stresses because it can affect the resistance mechanisms (i.e., physical, chemical or chemical / 
biochemical mechanisms) of the host plant against herbivory (Marschner, 2012). In this sense, potassium $(\mathrm{K})$ is an essential nutrient that helps plants defend against insects by favoring the development of stronger cell walls (Wang et al., 2013). On the other hand, calcium (Ca) also plays a role in plant defense mechanisms against insect herbivory, as it acts as a secondary marker under biotic stress and helps reinforce the cell walls and tissues (Marschner, 2012; War et al., 2012). Consequently, calcium foliar applications have been used as a tool to improve plant tolerance to insects (Hua et al., 2015).

The beneficial effects of silicon $(\mathrm{Si})$ application have been widely reported on crop growth and yield (Marschner, 2012). Likewise, silicon fertilization has shown to be an effective method to control herbivorous arthropods (Reynolds et al., 2016). The effectiveness of silicon against insect attacks is caused by the reduced digestibility and increased hardness and roughness of plant tissues, which is caused by the deposition of this mineral (Reynolds et al., 2009). In this aspect, foliar applications of Si reduced the population of thrips in tomato plants (Almeida et al., 2009).

D. citri was officially reported in Colombia in 2007 (Santivañez et al., 2013). However, an alert of phytosanitary emergency was declared in Colombia during 2015 by the Colombian Agricultural Institute (ICA) due to the presence of $D$. citri adults that were infected with the HLB disease bacterium, and the ICA also declared a national phytosanitary emergency due to this disease in the current year, i.e., 2017 (ICA 2015, 2017). For this reason, it is imperative to develop complementary strategies that strengthen the tolerance response in plants. Additionally, strategies that prevent psyllid feeding and ultimately affect its population dynamics should also be developed, with the purpose of preventing the acquisition and dissemination of the disease. Therefore, the objective of the present study was to evaluate the foliar and soil application of nutrients (i.e., calcium, potassium and silicon) that influence the mechanisms of plant resistance against the attack of arthropods.

\section{Materials and Methods}

\section{General experimental conditions}

Two separate experiments were carried out between November 2015 and January 2016 in the 'Parcela 3' (latitude: 4034’09.1" N, longitude: 7441'39.3” O, altitude: 313 asl) and 'Rochelita' (latitude: 4³3'07.6' N, longitude: $74^{\circ} 44^{\prime} 45.6^{\prime \prime} \mathrm{O}$, altitude: 263 asl) farms, which are located in the municipality of Jerusalén in the Department of Cundinamarca in Colombia. The environmental conditions are summarized in Fig. 1. Four-year-old and 2year-old Tahiti lime (Citrus latifolia Tanaka) trees grafted on 'Citrumelo CPB 4475' (Poncirus trifoliata $\times$ Citrus paradisi) were used in both farms, respectively. Additionally, all trees were spaced $6 \mathrm{~m} \times 4 \mathrm{~m}$.

The treatments performed in both farms included the following: 1) control trees (i.e., trees without nutrient or insecticide application); 2) trees treated with a neonicotinoid insecticide, clothianidin (Dantotsu ${ }^{\circ} 50$ WG; Arysta LifeScience, Colombia), at a dose of $50 \mathrm{~g}$ active ingredient (a.i.) per ha. Clothianidin was used because it has been reported in management programs for $D$. citri (Boina and Bloomquist, 2015); 3) trees treated with foliar $\mathrm{KNO}_{3}$ sprays at a dose of $3 \mathrm{~g}$ per $\left.\mathrm{L} \mathrm{H}_{2} \mathrm{O} ; 4\right)$ foliar applications of potassium silicate (Nitrosil-K, Agromil, Colombia) at a dose of $2 \mathrm{~mL} \cdot \mathrm{L}$; 5) foliar treatment of trees with calcium (Poliquel ${ }^{\circ}$ Calcio, Arysta, Colombia) at a dose of $3 \mathrm{~mL} \cdot \mathrm{L}$; and 6) soil $\mathrm{K}$ and $\mathrm{Si}$ applications were performed by applying $\mathrm{KNO}_{3}$ (Nitrato de Potasio MF, Microfertisa, Colombia) and $\mathrm{K}_{2} \mathrm{SiO}_{3}$ (Siliceo-K, Agromil, Colombia) at a dose of $1 \mathrm{~kg}$ of commercial fertilizer per tree, respectively.

In general, foliar nutrient sprays were applied at 0 and 4 weeks after treatment (WAT) and started between 07:00 and 09:00 h. Sprays were applied using a pesticide backpack sprayer with a volume of application of $2 \mathrm{~L}$ per tree, and both the upper and lower surfaces were wetted. In addition, foliar sprays were carried out without surfactants. Regarding soil applications, these treatments were performed at the beginning of the trial (i.e., 0 WAT) by incorporating the fertilizer into the soil around the canopy area. The experiments lasted 9 weeks. Finally, each experimental unit (i.e., treatment) included three trees on each farm. In addition, the experimental design was laid in a randomized block design, and each treatment was replicated four times (i.e., four blocks). In total, twelve trees received each treatment on each farm.
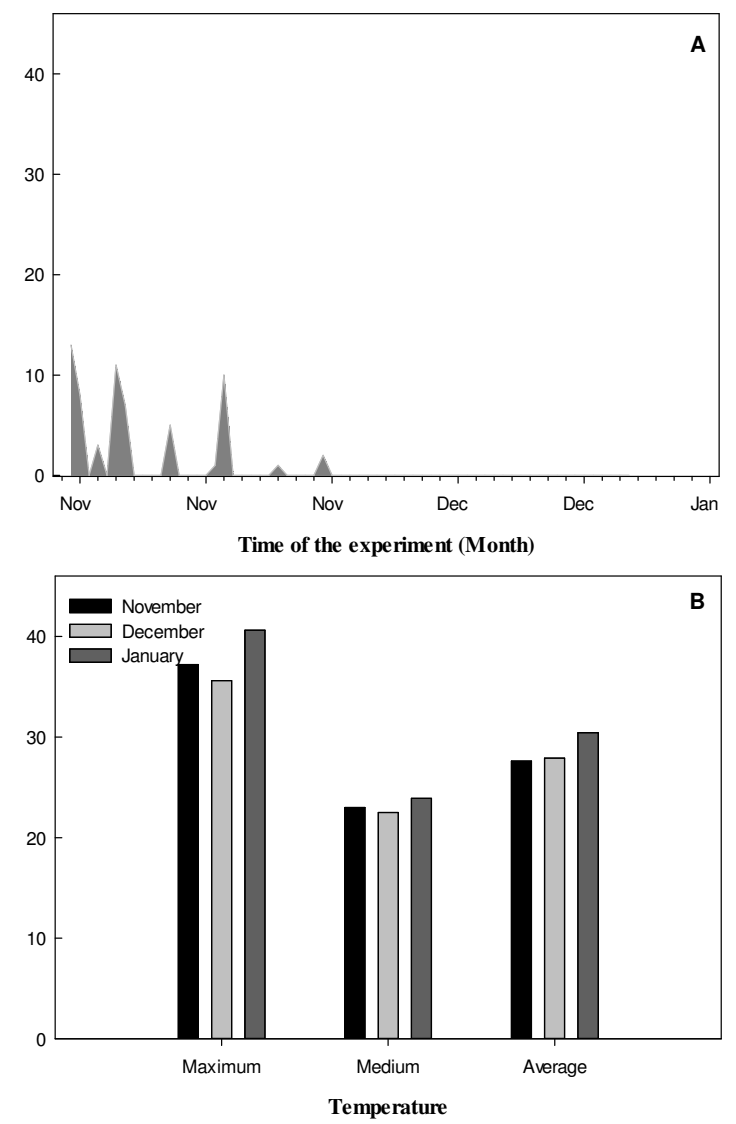

Fig. 1. Rainfall records (A) and maximum, minimum and average temperatures (B) for nutrient experiments placed in Jerusalén. Data were obtained from a nearby weather station (latitude: 4056'18'06" N; longitude: 7470'23.99"; altitude: 297) of the Institute of Hydrology, Meteorology and Environmental Studies of Colombia (IDEAM) 
548

\section{Population dynamics of D. citri}

The methodology described by Sétamou et al. (2008) was used to estimate the population variability of $D$. citri. The relative population levels of adults, eggs and nymphs per flush shoot were estimated in three trees per experimental unit by counting the number of each (adults, eggs, and nymphs) on each flush shoot. Four flush shoots were selected for each tree at each cardinal point of the tree. Adults were counted by visual examination, while a $15 x$ handheld magnifying lens was used to count the number of visible eggs and nymphs per flush shoot. The populations of psyllids per flush were estimated weekly between 0 and 8 WAT.

\section{Efficacy percentage}

The procedure described by Henderson and Tilton (1955) was used to evaluate the effect of each treatment on the development of $D$. citri populations. Overall, the efficacy percentage of each treatment was determined by the following formula:

$$
\% \text { Efficacy }=100 \times\left(1-\frac{(\text { Ta } \times C b)}{(\text { Tb } \times C a)}\right)
$$

Where $\mathbf{T}$ is the treated population, $\mathbf{C}$ is the control population, $\mathbf{a}$ is the population after the treatment, and $\mathbf{b}$ is the population before the treatment.

\section{Statistical design}

Data from a series of experiments performed at several sites are generally analysed together at the end of each crop experiment to evaluate the treatment $\times$ site interaction effect and the average effects of the treatments over homogeneous sites (Gomez and Gomez 1984). Therefore, a statistical design in series of experiments with analysis in different localities was used in the present study.
Additionally, untreated trees were included in each block, and they served as controls. The experimental unit was composed of three trees surrounded by guard trees, and each treatment was replicated four times (i.e., four blocks). Subsequently, an ANOVA was performed for each treatment. To analyze the population of $D$. citri, the data were $\log$-transformed $(\log X+1)$ because they were not normally distributed. Likewise, the data expressed as percentages were transformed using the arcsine formula. Tukey's test of mean comparison was used when significant differences were observed in the ANOVA. Data were analyzed using the statistical program Statistix (Version 9, Tallahassee, FL).

\section{Results and Discussion}

The population dynamics of $D$. citri adults mainly showed significant differences between farms from 1 to 4 WAT, obtaining the highest number of individuals at the "la Rochelita" farm (data not shown) (Table 1). Regarding treatments, differences were only observed in adults at 7 WAT where foliar calcium and silicon sprays had the lowest number of individuals (1.13 per flush) compared to the untreated trees (3.13 per flush). Also, treatments affected nymphs' population at 4 and 5 WAT, respectively. In this respect, a smaller number of nymphs were found in trees treated with clothianidin (1.63 individuals per flush) at 4 WAT than the other treatments. However, foliar calcium sprays had the lowest number of individuals per flush (1.25) compared to the control (6.00) and chemical insecticide (2.63) at 5 WAT (Table 2). Finally, either soil or foliar silicon application caused a negative effect on D. citri oviposition. Tahiti lime trees treated with silicon and insecticide had fewer eggs than did the trees in the other treatments at 1,3, 4, 5 and 6 WAT (Table 3).

Table 1. Population dynamics of Diaphorina citri adults under different application treatments of potassium, calcium and silicon in Tahiti lime trees

\begin{tabular}{|c|c|c|c|c|c|c|c|c|}
\hline \multirow{2}{*}{ Treatment } & \multicolumn{8}{|c|}{ Weeks After Treatments ${ }^{y}$} \\
\hline & 1 & 2 & 3 & 4 & 5 & 6 & 7 & 8 \\
\hline Control & $1.00 \pm 0.38^{x}$ & $0.75 \pm 0.25$ & $0.88 \pm 0.40$ & $1.5 \pm 0.93$ & $1.38 \pm 0.63$ & $1.33 \pm 0.37$ & $3.13 \pm 0.30 \mathrm{a}^{2}$ & $0.75 \pm 0.75$ \\
\hline Clothianidin & $0.38 \pm 0.26$ & $0.38 \pm 0.18$ & $0.75 \pm 0.41$ & $0.5 \pm 0.27$ & $0.63 \pm 0.26$ & $0.88 \pm 0.30$ & $1.38 \pm 0.50 \mathrm{ab}$ & $0.38 \pm 0.26$ \\
\hline $\mathrm{KNO}_{3}$-Foliar & $0.62 \pm 0.18$ & $0.88 \pm 0.29$ & $1.30 \pm 0.45$ & $1.25 \pm 0.49$ & $1.13 \pm 0.29$ & $0.88 \pm 0.30$ & $1.63 \pm 0.50 \mathrm{ab}$ & $0.25 \pm 0.25$ \\
\hline $\mathrm{KNO}_{3}$-Edaphic & $0.50 \pm 0.18$ & $0.13 \pm 0.13$ & $0.75 \pm 0.25$ & $0.75 \pm 0.25$ & $0.75 \pm 0.41$ & $0.88 \pm 0.30$ & $2.75 \pm 0.75 \mathrm{ab}$ & $0.25 \pm 0.25$ \\
\hline Calcium-Foliar & $0.75 \pm 0.31$ & $0.38 \pm 0.18$ & $0.63 \pm 0.27$ & $0.63 \pm 0.42$ & $1.00 \pm 0.27$ & $0.50 \pm 0.27$ & $1.12 \pm 0.30 \mathrm{~b}$ & $0.25 \pm 0.25$ \\
\hline Silicon-Foliar & $0.50 \pm 0.27$ & $0.63 \pm 0.32$ & $1.87 \pm 0.55$ & $0.88 \pm 0.51$ & $0.88 \pm 0.29$ & $0.63 \pm 0.26$ & $1.13 \pm 0.39 b$ & $0.00 \pm 0.00$ \\
\hline \multirow[t]{2}{*}{ Silicon-Edaphic } & $0.50 \pm 0.27$ & $0.50 \pm 0.27$ & $0.75 \pm 0.31$ & $0.50 \pm 0.38$ & $0.88 \pm 0.13$ & $0.25 \pm 0.17$ & $1.63 \pm 0.38 \mathrm{ab}$ & $0.25 \pm 0.25$ \\
\hline & \multicolumn{8}{|c|}{ Analysis of variance } \\
\hline Farm $(F)$ & $\begin{array}{l}F=30.16 \\
\mathrm{df}=1,55 \\
P=0.000\end{array}$ & $\begin{array}{c}F=14.52 ; \mathrm{df} \\
=1,55 ; \\
P=0.000\end{array}$ & $\begin{array}{l}F=8.75 \\
\mathrm{df}=1,55 \\
P=0.005\end{array}$ & $\begin{array}{l}F=26.60 \\
\mathrm{df}=1,55 \\
P=0.000\end{array}$ & $\begin{array}{l}F=0.03 \\
\mathrm{df}=1,55 \\
P=0.855\end{array}$ & $\begin{array}{l}F=11.65 \\
\mathrm{df}=1,55 \\
P=0.001\end{array}$ & $\begin{array}{l}F=2.15 \\
\mathrm{df}=1,55 \\
P=0.149\end{array}$ & $\begin{array}{l}F=2.02 \\
\mathrm{df}=1,55 \\
P=0.163\end{array}$ \\
\hline Treatments $(\mathrm{T})$ & $\begin{array}{l}F=0.81 \\
\mathrm{df}=6,55 \\
P=0.569\end{array}$ & $\begin{array}{l}F=1.46 \\
\mathrm{df}=6,55 \\
P=0.216\end{array}$ & $\begin{array}{l}F=1.04 \\
\mathrm{df}=6,55 \\
P=0.415\end{array}$ & $\begin{array}{l}F=0.68 \\
\mathrm{df}=6,55 \\
P=0.666\end{array}$ & $\begin{array}{l}F=0.43 \\
\mathrm{df}=6,55 \\
P=0.855\end{array}$ & $\begin{array}{l}F=1.44 \\
\mathrm{df}=6,55 \\
P=0.222\end{array}$ & $\begin{array}{l}F=2.90 \\
\mathrm{df}=6,55 \\
P=0.018\end{array}$ & $\begin{array}{l}F=0.27 \\
\mathrm{df}=6.55 \\
P=0.948\end{array}$ \\
\hline $\mathrm{F} \times \mathrm{T}$ & $\begin{array}{l}F=0.47 \\
\mathrm{df}=6,55 \\
P=0.830\end{array}$ & $\begin{array}{l}F=30.16 \\
\mathrm{df}=6,55 \\
P=0.153\end{array}$ & $\begin{array}{l}F=0.51 \\
\mathrm{df}=6,55 \\
P=0.794\end{array}$ & $\begin{array}{l}F=0.56 \\
\mathrm{df}=6,55 \\
P=0.762\end{array}$ & $\begin{array}{l}F=0.62 \\
\mathrm{df}=6.55 \\
P=0.709\end{array}$ & $\begin{array}{l}F=0.69 \\
\mathrm{df}=6,55 \\
P=0.657\end{array}$ & $\begin{array}{l}F=1.40 \\
\mathrm{df}=6,55 \\
P=0.239\end{array}$ & $\begin{array}{l}F=0.66 \\
\mathrm{df}=6.55 \\
P=0.682\end{array}$ \\
\hline
\end{tabular}

${ }^{2}$ Means with different letters represent statistically significant differences according to the Tukey test $(\mathrm{P} \leq 0.05)$.

y Treatment applications were conducted at 1 and 4 weeks after the beginning of the experiment.

${ }^{\mathrm{x}}$ Standard error of each media. 
Table 2. Population dynamics of Diaphorina citri nymphs under different application treatments of potassium, calcium and silicon in Tahiti lime trees

\begin{tabular}{|c|c|c|c|c|c|c|c|c|}
\hline \multirow{2}{*}{ Treatment } & \multicolumn{8}{|c|}{ Weeks After Treatment ${ }^{y}$} \\
\hline & 1 & 2 & 3 & 4 & 5 & 6 & 7 & 8 \\
\hline Control & $6.50 \pm 1.78^{x}$ & $9.25 \pm 3.10$ & $6.88 \pm 2.38$ & $7.63 \pm 1.59 a^{2}$ & $6.00 \pm 1.73 \mathrm{ab}$ & $7.63 \pm 2.03$ & $13.50 \pm 4.43$ & $29.87 \pm 6.96$ \\
\hline $\begin{array}{l}\text { Chemical Control } \\
\text { (Clothianidin) }\end{array}$ & $2.25 \pm 0.79$ & $2.38 \pm 0.96$ & $1.75 \pm 0.59$ & $1.63 \pm 0.53 b$ & $2.63 \pm 0.65 \mathrm{ab}$ & $2.88 \pm 0.66$ & $4.4 \pm 1.40$ & $15.25 \pm 5.56$ \\
\hline $\mathrm{KNO}_{3}$-Foliar & $2.62 \pm 0.75$ & $4.38 \pm 1.23$ & $1.75 \pm 0.83$ & $5.38 \pm 1.17 \mathrm{ab}$ & $6.88 \pm 1.54 \mathrm{a}$ & $4.12 \pm 1.41$ & $8.63 \pm 2.89$ & $12.87 \pm 3.68$ \\
\hline $\mathrm{KNO}_{3}$-Edaphic & $4.75 \pm 1.49$ & $5.25 \pm 2.05$ & $3.38 \pm 1.13$ & $5.13 \pm 1.78 \mathrm{ab}$ & $2.75 \pm 0.52 \mathrm{ab}$ & $3.50 \pm 1.05$ & $3.00 \pm 0.92$ & $14.87 \pm 5.16$ \\
\hline Calcium-Foliar & $4.38 \pm 1.25$ & $4.00 \pm 1.41$ & $3.13 \pm 1.64$ & $3.00 \pm 1.90 \mathrm{ab}$ & $1.25 \pm 0.52 \mathrm{~b}$ & $2.13 \pm 1.10$ & $3.34 \pm 1.22$ & $10.00 \pm 4.92$ \\
\hline Silicon-Foliar & $5.38 \pm 2.33$ & $3.38 \pm 1.25$ & $1.75 \pm 1.19$ & $4.13 \pm 1.46 \mathrm{ab}$ & $4.80 \pm 1.27 \mathrm{ab}$ & $4.88 \pm 1.18$ & $6.63 \pm 2.06$ & $19.75 \pm 8.73$ \\
\hline \multirow[t]{2}{*}{ Silicon-Edaphic } & $2.38 \pm 0.65$ & $5.25 \pm 2.52$ & $3.38 \pm 1.46$ & $2.50 \pm 0.80 \mathrm{ab}$ & $2.00 \pm 0.80 \mathrm{ab}$ & $3.63 \pm 1.47$ & $5.37 \pm 1.68$ & $17.25 \pm 4.83$ \\
\hline & \multicolumn{8}{|c|}{ Analysis of variance } \\
\hline Farm $(F)$ & $\begin{array}{l}F=30.85 \\
\mathrm{df}=1,55 \\
P=0.000\end{array}$ & $\begin{array}{c}F=31.52 ; \mathrm{df} \\
\quad=1,55 ; \\
P=0.000\end{array}$ & $\begin{aligned} F & =18.54 \\
\mathrm{df} & =1,55 ; P \\
& =0.000\end{aligned}$ & $\begin{array}{l}F=1.07 \\
\mathrm{df}=1,55 \\
P=0.307\end{array}$ & $\begin{array}{l}F=3.78 \\
\mathrm{df}=1,55 \\
P=0.058\end{array}$ & $\begin{array}{l}F=3.10 \\
\mathrm{df}=1,55 \\
P=0.085\end{array}$ & $\begin{array}{l}F=3.59 \\
\mathrm{df}=1,55 \\
P=0.065\end{array}$ & $\begin{array}{l}F=1.45 \\
\mathrm{df}=1,55 \\
P=0.236\end{array}$ \\
\hline Treatments $(\mathrm{T})$ & $\begin{array}{l}F=1.69 \\
\mathrm{df}=6,55 \\
P=0.147\end{array}$ & $\begin{array}{l}F=1.11 \\
\mathrm{df}=6,55 \\
P=0.374\end{array}$ & $\begin{array}{l}F=1.42 \\
\mathrm{df}=6,55 \\
P=0.229\end{array}$ & $\begin{array}{l}F=2.66 \\
\mathrm{df}=6,55 \\
P=0.028\end{array}$ & $\begin{array}{l}F=2.86 \\
\mathrm{df}=6,55 \\
P=0.019\end{array}$ & $\begin{array}{l}F=1.75 \\
\mathrm{df}=6,55 \\
P=0.132\end{array}$ & $\begin{array}{l}F=1.94 \\
\mathrm{df}=6,55 \\
P=0.096\end{array}$ & $\begin{array}{l}F=1.24 \\
\mathrm{df}=6,55 \\
P=0.305\end{array}$ \\
\hline $\mathrm{F} \times \mathrm{T}$ & $\begin{aligned} F & =1.74 ; \\
\mathrm{df} & =6,55 ; P \\
& =0.134\end{aligned}$ & $\begin{array}{l}F=1.23 \\
\mathrm{df}=6,55 \\
P=0.308\end{array}$ & $\begin{array}{l}F=0.85 \\
\mathrm{df}=6,55 \\
P=0.538\end{array}$ & $\begin{array}{l}F=1.22 \\
\mathrm{df}=6,55 \\
P=0.313\end{array}$ & $\begin{array}{l}F=0.45 \\
\mathrm{df}=6,55 \\
P=0.837\end{array}$ & $\begin{array}{l}F=0.75 \\
\mathrm{df}=6,55 \\
P=0.609\end{array}$ & $\begin{array}{l}F=0.89 \\
\mathrm{df}=6,55 \\
P=0.508\end{array}$ & $\begin{array}{l}F=0.51 \\
\mathrm{df}=6,55 \\
P=0.796\end{array}$ \\
\hline
\end{tabular}

$\mathrm{z}$ Means with different letters represent statistically significant differences according to the Tukey test $(\mathrm{P} \leq 0.05)$.

y Treatment applications were conducted at 1 and 4 weeks after the beginning of the experiment.

$\mathrm{x}$ Standard error of each media.

Table 3. Population dynamics of Diaphorina citri eggs under different application treatments of potassium, calcium and silicon in Tahiti lime trees

\begin{tabular}{|c|c|c|c|c|c|c|c|c|}
\hline \multirow{2}{*}{ Treatment } & \multicolumn{8}{|c|}{ Weeks After Treatment ${ }^{y}$} \\
\hline & 1 & 2 & 3 & 4 & 5 & 6 & 7 & 8 \\
\hline Control & $8.38 \pm 1.90 a^{z}$ & $6.75 \pm 2.68^{x}$ & $10.25 \pm 1.97 \mathrm{a}$ & $10.88 \pm 2.09 \mathrm{a}$ & $11.13 \pm 1.81 \mathrm{a}$ & $13.38 \pm 2.67 \mathrm{a}$ & $22.00 \pm 5.61$ & $29.88 \pm 8.66$ \\
\hline $\begin{array}{l}\text { Chemical Control } \\
\text { (Clothianidin) }\end{array}$ & $1.75 \pm 0.41 \mathrm{~b}$ & $2.75 \pm 0.52$ & $1.75 \pm 0.36 \mathrm{~b}$ & $2.38 \pm 0.37 b$ & $5.13 \pm 1.20 \mathrm{~b}$ & $4.63 \pm 0.94 \mathrm{~b}$ & $8.38 \pm 2.18$ & $11.75 \pm 2.41$ \\
\hline $\mathrm{KNO}_{3}$-Foliar & $3.75 \pm 1.16 \mathrm{ab}$ & $2.87 \pm 0.55$ & $3.75 \pm 0.95 \mathrm{ab}$ & $3.50 \pm 0.80 \mathrm{~b}$ & $5.50 \pm 1.08 b$ & $6.25 \pm 1.11 \mathrm{~b}$ & $7.88 \pm 1.70$ & $9.25 \pm 2.12$ \\
\hline $\mathrm{KNO}_{3}$-Edaphic & $6.00 \pm 2.40 \mathrm{ab}$ & $4.62 \pm 1.05$ & $5.13 \pm 1.41 \mathrm{ab}$ & $3.75 \pm 0.64 b$ & $8.50 \pm 1.83 \mathrm{ab}$ & $8.75 \pm 1.48 \mathrm{ab}$ & $12.00 \pm 5.33$ & $23.63 \pm 7.32$ \\
\hline Calcium-Foliar & $3.5 \pm 0.80 \mathrm{ab}$ & $3.25 \pm 1.16$ & $5.13 \pm 1.28 \mathrm{ab}$ & $4.63 \pm 1.20 \mathrm{~b}$ & $5.40 \pm 1.40 \mathrm{~b}$ & $8.75 \pm 2.05 \mathrm{ab}$ & $8.75 \pm 1.70$ & $8.63 \pm 2.12$ \\
\hline Silicon-Foliar & $2.5 \pm 0.56 b$ & $3.50 \pm 0.50$ & $3.25 \pm 1.04 b$ & $3.50 \pm 0.59 \mathrm{~b}$ & $6.50 \pm 1.83 \mathrm{ab}$ & $5.75 \pm 1.16 \mathrm{~b}$ & $6.75 \pm 1.39$ & $10.13 \pm 3.20$ \\
\hline \multirow[t]{2}{*}{ Silicon-Edaphic } & $3.5 \pm 1.28 \mathrm{~b}$ & $2.38 \pm 0.53$ & $3.50 \pm 0.63 b$ & $4.25 \pm 0.83 b$ & $5.25 \pm 0.37 b$ & $4.75 \pm 0.65 b$ & $9.13 \pm 1.65$ & $11.13 \pm 2.85$ \\
\hline & \multicolumn{8}{|c|}{ Analysis of variance } \\
\hline Farm (F) & $\begin{array}{l}F=36.52 \\
\mathrm{df}=1,55 \\
P=0.000\end{array}$ & $\begin{array}{l}F=12.84 \\
\mathrm{df}=1,55 \\
P=0.000\end{array}$ & $\begin{array}{l}F=14.68 \\
\mathrm{df}=1,55 \\
P=0.000\end{array}$ & $\begin{array}{l}F=17.78 \\
\mathrm{df}=1,55 \\
P=0.000\end{array}$ & $\begin{array}{l}F=9.14 \\
\mathrm{df}=1,55 \\
P=0.004\end{array}$ & $\begin{array}{l}F=33.78 \\
\mathrm{df}=1,55 \\
P=0.000\end{array}$ & $\begin{array}{l}F=31.68 \\
\mathrm{df}=1,55 \\
P=0.000\end{array}$ & $\begin{array}{l}F=10.54 \\
\mathrm{df}=1,55 \\
P=0.002\end{array}$ \\
\hline Treatments $(T)$ & $\begin{array}{l}F=4.33 ; \\
\mathrm{df}=6,55 ; \\
P=0.001\end{array}$ & $\begin{array}{l}F=1.62 ; \\
\mathrm{df}=6,55 ; \\
P=0.165\end{array}$ & $\begin{array}{l}F=3.54 \\
\mathrm{df}=6.55 \\
P=0.006\end{array}$ & $\begin{array}{l}F=5.86 \\
\mathrm{df}=6,55 \\
P=0.000\end{array}$ & $\begin{array}{c}F=2.16 \\
\mathrm{df}=6,55 \\
P=0.0458\end{array}$ & $\begin{array}{l}F=5.20 \\
\mathrm{df}=6.55 \\
P=0.000\end{array}$ & $\begin{array}{l}F=1.44 \\
\mathrm{df}=6.55 \\
P=0.222\end{array}$ & $\begin{array}{l}F=1.20 \\
\mathrm{df}=6,55 \\
P=0.3324\end{array}$ \\
\hline $\mathrm{F} \times \mathrm{T}$ & $\begin{array}{l}F=1.77 ; \\
\text { df }=6,55 ; \\
P=0.128\end{array}$ & $\begin{array}{l}F=0.90 \\
\mathrm{df}=6.55 \\
P=0.503\end{array}$ & $\begin{array}{l}F=0.84 \\
\mathrm{df}=6.55 \\
P=0.547\end{array}$ & $\begin{array}{l}F=0.72 \\
\mathrm{df}=6.55 \\
P=0.632\end{array}$ & $\begin{array}{l}F=0.62 \\
\mathrm{df}=6.55 \\
P=0.713\end{array}$ & $\begin{array}{l}F=1.65 \\
\mathrm{df}=6,55 \\
P=0.158\end{array}$ & $\begin{array}{l}F=1.04 \\
\mathrm{df}=6,55 \\
P=0.414\end{array}$ & $\begin{array}{l}F=1.33 \\
\mathrm{df}=6.55 \\
P=0.264\end{array}$ \\
\hline
\end{tabular}

${ }^{\mathrm{z}}$ Means with different letters represent statistically significant differences according to the Tukey test $(\mathrm{P} \leq 0.05)$.

${ }^{y}$ Treatment applications were conducted at 1 and 4 weeks after the beginning of the experiment.

${ }^{\mathrm{x}}$ Standard error of each media

When analyzing the data as the mean and total number of individuals, it was observed that the insecticide treatment and the soil silicon application showed the lowest number of nymphs (mean and accumulated nymphs per flush were 3 and 23, respectively) in comparison to control trees (mean and accumulated nymphs per flush were 7 and 62, respectively) (Table 4). The efficacy percentage of the different treatments was evaluated from 2 to 8 WAT for both the nymphs and eggs of $D$. citri (Table 5). Significant differences between treatments $(\mathrm{P} \leq 0.05)$ were only obtained at 6 WATin nymphs where the greatest efficacy was obtained in trees treated with $\mathrm{KNO}_{3}$ sprays (41\%) rather than in trees treated with foliar calcium applications (24\%). On the other hand, no differences were observed on the efficacy percentage in ACP oviposition among treatments. 
550

Table 4: Individual means and total individuals of Diaphorina citri under different application treatments of potassium, calcium and silicon in Tahiti lime trees

\begin{tabular}{|c|c|c|c|c|c|c|}
\hline \multirow{3}{*}{ Treatment } & \multicolumn{3}{|c|}{ Individual Means } & \multicolumn{3}{|c|}{ Total Individuals } \\
\hline & \multicolumn{3}{|c|}{ Development Stage } & \multicolumn{3}{|c|}{ Development Stage } \\
\hline & Adults & Nymphs & Eggs & Adults & Nymphs & Eggs \\
\hline Control & $1.36 \pm 0.35^{y}$ & $7.72 \pm 1.70 a^{z}$ & $11.61 \pm 1.80 \mathrm{a}$ & $10.87 \pm 2.81$ & $61.75 \pm 13.64 \mathrm{a}$ & $92.88 \pm 14.46 \mathrm{a}$ \\
\hline Clothianidin & $0.77 \pm 0.25$ & $2.85 \pm 0.71 \mathrm{~b}$ & $4.45 \pm 0.7 b$ & $6.13 \pm 2.05$ & $22.75 \pm 5.65 b$ & $35.63 \pm 5.68 \mathrm{~b}$ \\
\hline $\mathrm{KNO}_{3}$-Foliar & $1.07 \pm 0.27$ & $4.81 \pm 0.80 \mathrm{ab}$ & $4.92 \pm 0.88 b$ & $8.63 \pm 2.12$ & $38.87 \pm 6.44 \mathrm{ab}$ & $39.38 \pm 7.11 \mathrm{~b}$ \\
\hline $\mathrm{KNO}_{3}$ Edaphic & $1.03 \pm 0.14$ & $4.41 \pm 0.65 \mathrm{ab}$ & $7.13 \pm 1.13 b$ & $8.25 \pm 1.16$ & $35.29 \pm 5.20 \mathrm{ab}$ & $57.00 \pm 11.12 b$ \\
\hline Calcium-Foliar & $0.78 \pm 0.21$ & $3.98 \pm 1.01 \mathrm{ab}$ & $5.91 \pm 1.34 b$ & $6.25 \pm 1.76$ & $31.87 \pm 8.04 \mathrm{ab}$ & $47.25 \pm 10.78 b$ \\
\hline Silicon-Foliar & $0.92 \pm 0.30$ & $4.83 \pm 1.41 \mathrm{ab}$ & $5.00 \pm 0.85 b$ & $7.38 \pm 2.43$ & $38.62 \pm 11.28 \mathrm{ab}$ & $40.00 \pm 6.81 \mathrm{~b}$ \\
\hline Silicon-Edaphic & $0.72 \pm 0.21$ & $3.33 \pm 0.88 b$ & $4.85 \pm 0.74 b$ & $5.57 \pm 1.75$ & $26.63 \pm 7.06 \mathrm{~b}$ & $38.75 \pm 2.75 b$ \\
\hline Farm $(F)$ & $\begin{array}{l}F=29.42 \\
\mathrm{df}=1,55 \\
P=0.000\end{array}$ & $\begin{array}{l}F=20.78 \\
\mathrm{df}=1,55 \\
P=0.000\end{array}$ & $\begin{array}{l}F=85.95 \\
\mathrm{df}=1,55 \\
P=0.000\end{array}$ & $\begin{array}{l}F=27.99 \\
\mathrm{df}=1,55 \\
P=0.000\end{array}$ & $\begin{array}{l}F=16.62 \\
\mathrm{df}=1,55 \\
P=0.000\end{array}$ & $\begin{array}{l}F=79.24 \\
\mathrm{df}=1,55 \\
P=0.000\end{array}$ \\
\hline $\begin{array}{l}\text { Treatments } \\
\qquad(\mathrm{T})\end{array}$ & $\begin{array}{l}F=1.19 \\
\mathrm{df}=6,55 \\
P=0.330\end{array}$ & $\begin{array}{l}F=2.92 \\
\mathrm{df}=6,55 \\
P=0.018\end{array}$ & $\begin{array}{l}F=9.52 \\
\mathrm{df}=6,55 \\
P=0.000\end{array}$ & $\begin{array}{l}F=1.06 \\
\mathrm{df}=6,55 \\
P=0.403\end{array}$ & $\begin{array}{l}F=2.32 \\
\mathrm{df}=6.55 \\
P=0.046\end{array}$ & $\begin{array}{l}F=8.42 ; \\
\mathrm{df}=6,55 ; \\
P=0.000\end{array}$ \\
\hline $\mathrm{F} \times \mathrm{T}$ & $\begin{array}{l}F=0.56 \\
\mathrm{df}=6.55 \\
\mathrm{P}=0.757\end{array}$ & $\begin{array}{c}F=0.61 \\
\mathrm{df}=6.55 \\
P=0.721\end{array}$ & $\begin{array}{l}F=1.56 \\
\mathrm{df}=6,55 \\
P=0.182\end{array}$ & $\begin{array}{l}F=0.58 \\
\mathrm{df}=6.55 \\
\mathrm{P}=0.741\end{array}$ & $\begin{array}{l}F=0.50 \\
\mathrm{df}=6,55 \\
P=0.806\end{array}$ & $\begin{array}{l}F=1.49 ; \\
\mathrm{df}=6,55 ; \\
P=0.207\end{array}$ \\
\hline
\end{tabular}

${ }^{ }$Means with different letters represent statistically significant differences according to the Tukey test $(\mathrm{P} \leq 0.05)$.

${ }^{y}$ Standard error of each media.

Table 5. Summary of the efficacy percentage of leaves treated with potassium, calcium and silicon on nymphs and eggs of Diaphorina citri in Tahiti lime trees

\begin{tabular}{|c|c|c|c|c|c|c|c|}
\hline \multirow{3}{*}{ Treatment } & \multicolumn{7}{|c|}{ Weeks After Treatment ${ }^{y}$} \\
\hline & 2 & 3 & 4 & 5 & 6 & 7 & 8 \\
\hline & \multicolumn{7}{|c|}{ Nymphs } \\
\hline Insecticide & $69.07 \pm 4.20$ & $55.76 \pm 4.39$ & $36.60 \pm 3.97$ & $36.73 \pm 5.59$ & $35.28 \pm 5.95 \mathrm{ab}^{\mathrm{z}}$ & $31.13 \pm 2.37^{x}$ & $33.26 \pm 4.18$ \\
\hline $\mathrm{KNO}_{3}$-Foliar & $69.26 \pm 3.75$ & $64.05 \pm 4.43$ & $34.67 \pm 4.94$ & $41.91 \pm 5.98$ & $41.20 \pm 4.85 \mathrm{a}$ & $25.65 \pm 3.94$ & $36.30 \pm 4.93$ \\
\hline $\mathrm{KNO}_{3}$ Edaphic & $76.72 \pm 4.41$ & $61.09 \pm 3.99$ & $40.67 \pm 4.20$ & $48.00 \pm 8.92$ & $26.98 \pm 3.94 \mathrm{ab}$ & $27.23 \pm 4.33$ & $29.13 \pm 4.27$ \\
\hline Calcium Foliar & $74.35 \pm 5.10$ & $57.61 \pm 5.71$ & $40.06 \pm 4.70$ & $40.11 \pm 4.93$ & $24.12 \pm 4.32 \mathrm{~b}$ & $24.98 \pm 3.53$ & $33.83 \pm 4.12$ \\
\hline Silicon-Foliar & $72.27 \pm 4.14$ & $54.30 \pm 3.62$ & $39.56 \pm 4.78$ & $39.87 \pm 6.72$ & $36.07 \pm 6.10 \mathrm{ab}$ & $31.77 \pm 5.01$ & $35.60 \pm 6.66$ \\
\hline Silicon-Edaphic & $72.95 \pm 2.99$ & $64.90 \pm 4.38$ & $42.71 \pm 4.34$ & $40.64 \pm 5.25$ & $30.60 \pm 3.57 \mathrm{ab}$ & $37.82 \pm 6.03$ & $35.08 \pm 5.29$ \\
\hline Farm $(F)$ & $\begin{array}{l}F=4.38 \\
\mathrm{df}=1,47 \\
P=0.043\end{array}$ & $\begin{array}{l}F=2.87 \\
\mathrm{df}=1,47 \\
P=0.099\end{array}$ & $\begin{array}{l}F=0.18 \\
\mathrm{df}=1,47 \\
P=0.669\end{array}$ & $\begin{array}{c}F=85.88 \\
\mathrm{df}=1,47 \\
P=0.000\end{array}$ & $\begin{array}{c}F=23.93 \\
\mathrm{df}=1,47 \\
P=0.000\end{array}$ & $\begin{array}{c}F=4.03 \\
\mathrm{df}=1,47 \\
P=0.0522\end{array}$ & $\begin{array}{l}F=1.26 \\
\mathrm{df}=1,47 \\
P=0.268\end{array}$ \\
\hline $\begin{array}{l}\text { Treatments } \\
\qquad(\mathrm{T})\end{array}$ & $\begin{array}{l}F=0.59 \\
\mathrm{df}=5,47 \\
P=0.705\end{array}$ & $\begin{array}{l}F=0.94 \\
\mathrm{df}=5,47 \\
P=0.465\end{array}$ & $\begin{array}{l}F=0.40 \\
\mathrm{df}=5,47 \\
P=0.845\end{array}$ & $\begin{aligned} F & =1.06 \\
\mathrm{df} & =5,, 47 \\
P & =0.399\end{aligned}$ & $\begin{array}{l}F=2.50 \\
\mathrm{df}=5,47 \\
P=0.048\end{array}$ & $\begin{array}{l}F=1.40 \\
\mathrm{df}=5,47 \\
P=0.247\end{array}$ & $\begin{array}{l}F=0.24 \\
\mathrm{df}=5,47 \\
P=0.942\end{array}$ \\
\hline \multirow[t]{2}{*}{$\mathrm{F} \times \mathrm{T}$} & $\begin{array}{l}F=0.91 \\
\mathrm{df}=5,47 \\
\mathrm{P}=0.482\end{array}$ & $\begin{array}{c}F=0.18 \\
\mathrm{df}=5,47 \\
P=0.966\end{array}$ & $\begin{array}{c}F=0.31 \\
\mathrm{df}=5,47 \\
P=0.904\end{array}$ & $\begin{array}{l}F=1.60 \\
\mathrm{df}=5,47 \\
\mathrm{P}=0.184\end{array}$ & $\begin{array}{l}F=0.20 \\
\mathrm{df}=5,47 \\
\mathrm{P}=0.961\end{array}$ & $\begin{array}{c}F=1.95 \\
\mathrm{df}=5,47 \\
P=0.109\end{array}$ & $\begin{array}{l}F=0.42 ; \\
\mathrm{df}=5,47 \\
P=0.834\end{array}$ \\
\hline & \multicolumn{7}{|c|}{ Eggs } \\
\hline Insecticide & $58.67 \pm 4.14$ & $42.87 \pm 4.98$ & $42.77 \pm 4.40$ & $33.20 \pm 2.30$ & $40.41 \pm 3.11$ & $30.73 \pm 2.85$ & $39.53 \pm 4.65$ \\
\hline $\mathrm{KNO}_{3}$-Foliar & $64.73 \pm 5.97$ & $44.38 \pm 6.35$ & $51.52 \pm 3.63$ & $35.98 \pm 4.00$ & $36.67 \pm 2.50$ & $34.15 \pm 2.28$ & $38.71 \pm 2.45$ \\
\hline $\mathrm{KNO}_{3}$ Edaphic & $63.63 \pm 5.88$ & $43.36 \pm 4.46$ & $50.22 \pm 4.98$ & $35.38 \pm 3.62$ & $40.06 \pm 4.38$ & $35.35 \pm 2.49$ & $39.42 \pm 2.46$ \\
\hline Calcium Foliar & $59.85 \pm 6.04$ & $43.85 \pm 4.72$ & $49.16 \pm 4.07$ & $35.77 \pm 3.58$ & $34.46 \pm 2.16$ & $36.61 \pm 2.75$ & $36.81 \pm 2.79$ \\
\hline Silicon-Foliar & $54.86 \pm 4.97$ & $41.71 \pm 4.78$ & $44.35 \pm 4.24$ & $36.16 \pm 3.56$ & $37.51 \pm 3.65$ & $32.26 \pm 3.24$ & $36.06 \pm 2.52$ \\
\hline SiliconEdaphic & $59.63 \pm 7.05$ & $36.37 \pm 5.36$ & $48.01 \pm 5.35$ & $37.30 \pm 3.31$ & $36.33 \pm 2.00$ & $30.70 \pm 3.47$ & $40.51 \pm 4.24$ \\
\hline Farm $(\mathrm{F})$ & $\begin{array}{c}F=33.07 ; \mathrm{df}=1,47 \\
P=0.043\end{array}$ & $\begin{array}{c}F=0.00 ; \mathrm{df}=1,47 \\
P=0.955\end{array}$ & $\begin{array}{c}F=4.79 ; \mathrm{df}=1,47 \\
P=0.035\end{array}$ & $\begin{array}{c}F=0.14 ; \mathrm{df}=1,47 \\
P=0.715\end{array}$ & $\begin{array}{c}F=11.91 ; \mathrm{df}=1,47 ; \\
P=0.001\end{array}$ & $\begin{array}{c}F=2.54 ; \mathrm{df}=1,47 \\
P=0.120\end{array}$ & $\begin{array}{c}F=9.38 ; \mathrm{df}=1,47 ; \\
P=0.004\end{array}$ \\
\hline Treatments $(\mathrm{T})$ & $\begin{array}{c}F=0.72 ; \mathrm{df}=5,47 \\
P=0.612\end{array}$ & $\begin{array}{c}F=0.30 ; \mathrm{df}=5,47 \\
P=0.912\end{array}$ & $\begin{array}{c}F=0.61 ; \mathrm{df}=5,47 \\
P=0.696\end{array}$ & $\begin{array}{c}F=0.13 ; \mathrm{df}=5,, 47 \\
P=0.985\end{array}$ & $\begin{array}{c}F=0.64 ; \mathrm{df}=5,47 \\
P=0.674\end{array}$ & $\begin{array}{c}F=0.78 ; \mathrm{df}=5,47 \\
P=0.571\end{array}$ & $\begin{array}{c}F=0.29 ; \mathrm{df}=5,47 ; \\
P=0.918\end{array}$ \\
\hline $\mathrm{F} \times \mathrm{T}$ & $\begin{array}{c}F=1.19 ; \mathrm{df}=5,, 47 \\
P=0.331\end{array}$ & $\begin{array}{c}F=0.35 \mathrm{df}=5,, 47 \\
P=0.881\end{array}$ & $\begin{array}{c}F=0.56 ; \mathrm{df}=5,47 \\
P=0.728\end{array}$ & $\begin{array}{c}F=0.20 ; \mathrm{df}=5,, 47 \\
P=0.959\end{array}$ & $\begin{array}{c}F=0.48 ; \mathrm{df}=5,, 47 \\
P=0.787\end{array}$ & $\begin{array}{c}F=0.87 \mathrm{df}=5,, 47 \\
P=0.508\end{array}$ & $\begin{array}{c}F=0.45 ; \mathrm{df}=5,47 ; \\
P=0.8107\end{array}$ \\
\hline
\end{tabular}

${ }^{\bar{z}}$ Means with different letters represent statistically significant differences according to the Tukey test $(\mathrm{P} \leq 0.05)$.

y Treatment applications were conducted at 1 and 4 weeks after the beginning of the experiment.

${ }^{x}$ Standard error of each media. 
It has been widely documented that the application of nutrients, such as calcium, potassium and silicon, can improve crop protection against arthropods (Amtmann $e t$ al., 2008; Hua et al., 2015; Reynolds et al., 2016). The results obtained in the present study indicated that soil or foliar nutrients application (calcium, potassium and silicon) had positive effects by controlling the population of ACP nymphs and eggs. In this sense, foliar $\mathrm{KNO}_{3}$ applications showed a higher efficacy $(\approx 50 \%)$ at 4 WAT. Amtmann $e t$ al. (2008) reported that one of the beneficial effects of an adequate potassium supply is the reduced incidence of insects and mites. Additionally, Staley et al. (2011) mentioned that the type of fertilizer used could alter the plant-insect ratio, noting that the use of a potassium fertilizer reduced the infestation of Brevicoryne brassicae L. (Sternorrhyncha: Homoptera) in Brassica olearacea. The decrease in the incidence of ACP individuals caused by the use of $\mathrm{KNO}_{3}$ may be because the low $\mathrm{K}$ content in plants tends to increase the levels of soluble sugars, organic acids and amino acids, creating a beneficial environment for the development and feeding of insects (Amtmann et al., 2008).

Plants develop different strategies to protect themselves against herbivory. In this context, calcium plays an important role in recognizing invasive biotic agents in the cell membrane (Marschnner, 2012). A higher cytoplasmic $\mathrm{Ca}$ content has been reported in plants under stressful conditions because it can act as a secondary messenger (Nakata, 2015). Additionally, calcium is important because it provides stability to the bio-membranes, and a low content of this macronutrient favors the transport of sugars from the cytoplasm to the cell apoplast (Marschnner, 2012). In addition, calcium can be linked to oxalate and forms calcium oxalate crystals, which act as deterrents against arthropods (Schwachtje and Baldwin, 2008). The above information helps to understand why foliar Ca applications caused a low number of $D$. citri individuals in the present study (Table 4). Additionally, Dia de Almeida et al. (2008) observed that foliar calcium sprays also caused a decrease in populations of Thrips palmi (Thysanoptera: Thripidae) in Solanum melongena.

The use of silicon $(\mathrm{Si})$ has demonstrated its potential in the mitigation of biotic stresses (Reynolds et al., 2016). In the present study, the use of a silicate in both the soil and foliar applications also caused a decrease in the total number of ACP individuals (i.e., adults, nymphs and eggs) in Tahiti lime trees. Several authors have also reported the positive effects of Si applications in the control of Myzus persicae (Hemiptera: Aphididae) populations in zinnia (Ranger $e t$ al., 2009), Oligonychus sacchari (Acari: Tetranychidae) in sugarcane (Nikpay and Nejadian, 2014) and Aleurocanthus woglumi (Hemiptera: Aleyrodidade) in tangerine (Vieira et al., 2016). The silicon mechanisms involve in plant resistance to insect attacks can be due to this nutrient can enhance leaf roughness and hardness epidermis, reducing their digestibility. Additionally, Si can be deposited in a soluble form among leaf tissues, inhibiting sucking by the insect (Reynolds et al., 2009). ACP is mainly a phloemfeeding insect (Bonani et al., 2010), which means that when inserting its stylet, it must pass through many leaf layers (Garzo et al., 2011). Plants treated with $\mathrm{Ca}$ or $\mathrm{Si}$ can experience a hardening of their tissues, which prevents the insects from reaching the phloem. This can also increase the energy expenditure of the insects, which consequently affects the insect's survival and population growth (Marschner, 2012).

In summary, the results of the present study showed that foliar or soil mineral nutrients applications, such as $\mathrm{K}, \mathrm{Ca}$ and $\mathrm{Si}$, significantly reduced the total numbers of $D$. citri nymphs and eggs in Tahiti lime trees. Additionally, the use of these mineral nutrients had a similar efficacy with respect to clothianidin, indicating that they can enhance the resistance of the plant. This suggests that foliar applications of $\mathrm{K}, \mathrm{Ca}$ and $\mathrm{Si}$ should be considered within an integrated management program for $D$. citri as complementary tools to chemical control and that the application of these elements can generate physiological benefits in plants.

\section{References}

Almeida GD, Pratissoli D, Zanuncio J C, Vicentini VB, Holtz AM, Serrão JE (2009). Calcium silicate and organic mineral fertilizer increase the resistance of tomato plants to Frankliniella schultzei. Phytoparasitica 37:225-230.

Amtmann A, Troufflard S, Armengaud P (2008). The effect of potassium nutrition on pest and disease resistance in plants. Physiologia Plantarum 133:682-691.

Boina DR, Bloomquist JR (2015). Chemical control of the Asian citrus psyllid and of Huanglongbing disease in citrus. Pest Management Science 71(6):808-823.

BonaniJP, Fereres A, Appezzato-da-Gloria B, Garzo E, Miranda MP, Lopes, JRS (2010). Characterization of electrical penetration graphs of the Asian citrus psyllid, Diaphorina citri Kuwayama in sweet orange seedlings. Entomologia Experimentaliset Applicata 134(1):35-49.

Canales E, Coll Y, Hernandez I, Portieles R, Garcia MR, Lopez Y,... BorrasHidalgo O (2016). 'Candidatus Liberibacter asiaticus', causal agent of citrus Huanglongbing, is reduced by treatment with brassinosteroids. PloS One 11(1):e0146223.

Dia de Almeida G, Pratissoli D, Zanuncio JC, Vicentini VB, Holtz AM, Serrão JE (2008). Calcium silicate and organic mineral fertilizer applications reduce phytophagy by Thrips palmi Karny (Thysanoptera: Thripidae) on eggplants (Solanum melongena L.). Interciencia 33(11):835-838.

Garzo EG, Bonani JP, Lopes JRS, Fereres A (2011). Morphological description of the mouthparts of the Asian citrus psyllid, Diaphorina citri Kuwayama (Hemiptera: Psyllidae). Arthropod Structure and Development 41(1):79-86.

Gottwald TR (2010). Current epidemiological understanding of citrus huanglongbing. Annual Review of Phytopathology 48:119-139.

Gomez KA, Gomez AA (1984). Statistical procedures for agricultural research.John Wiley \& Sons, Toronto.

Hall DG, Richardson ML, Ammar ED, Halbert SE (2013). Asian citrus psyllid, Diaphorina citri, vector of citrus huanglongbing disease. Entomologia Experimentalis et Applicata 146:207-223.

Henderson CF, Tilton EW (1955). Tests with acaricides against the brown wheat mite.Journal of Economic Entomology 48:157-161.

Hua KH, Wang HC, Chung RS, Hsu JC (2015). Calcium carbonate 
552

nanoparticles can enhance plant nutrition and insect pest tolerance. Journal of PestScience 40(4):208-213.

ICA (2015). Resolución 2390 de 2015: "Por medio de la cual se declara el estado de emergencia fitosanitaria en el territorio nacional por la presencia de adultos de Diaphorina citri infectados con la bacteria de la enfermedad del HLB de los cítricos" [Resolution 2390 of 2015: "By means of which the state of phytosanitary emergency in the national territory is declared by the presence of adults of Diaphorina citri infected with the bacteria of the disease of the HLB of citrus fruits"]. Retrieved 2017 September 12 from http://www.ica.gov.co/Normatividad/.

ICA (2017). Resolución número 00007109 de 2017, por medio de la cual se declara el estado de emergencia fitosanitaria en el territorio nacional por la presencia de la enfermedad conocida como Huanglongbing (HLB) de los cítricos [Resolution number 00007109 of 2017 , by means of which the state of phytosanitary emergency in the national territory is declared by the presence of the disease known as Huanglongbing(HLB) of citrus fruits]. Retrieved 2017 September 12 from http://www.ica.gov.co/Normatividad/.

Jagoueix S, Bove JM, Garnier M (1994). The phloem-limited bacterium of greening disease of citrus is a member of alpha subdivision of Proteobacteria. International Journal of Systematic and Evolutionary Microbiology 44:379-386.

Marschner P (2012). Marschner's mineral nutrition of higher plants. Academic Press, London.

Nakata PA (2015). An assessment of engineered calcium oxalate crystal formation on plant growth and development as a step toward evaluating its use to enhance plant defense. Plos One 10(10):e0141982.

Nikpay A, Nejadian ES (2014). Field applications of silicon-based fertilizers against sugarcane yellow mite Oligonychus sacchari. Sugar Tech 16(3):319-324.

Orduz-RodríguezJO, Monroy HJ, Fischer G (2010). Phenological behavior of 'Arrayana' mandarin in the piedmont of the Meta department, Colombia. Agronomia Colombiana 28(1):63-70.

Orduz-Rodríguez JO, Garzon DC (2012). Alternate bearing and phenology of 'Valencia' orange (Citrus sinensis [L.] Osbeck) in the lowland wet tropics of Colombia. Corpoica Ciencia y Tecnologia Agropecuaria 13(2):136-144.

Pluke RWH, Qureshi JA, Stansly PA (2008). Citrus flushing patterns, Diaphorina citri (Hemiptera: Psyllidae) populations and parasitism by Tamarixia radiata (Hymenoptera: Eulophidae) in Puerto Rico. Florida Entomologist 91(1):36-42.
Ranger CM, Singh AP, Frantz JM, Canas L, Locke JC, Reding ME, Vorsa $\mathrm{N}$ (2009). Influence of silicon on resistance of Zinnia elegans to Myzus persicae (Hemiptera: Aphididae). Environmental Entomology 38(1):129-136.

Reynolds OL, Keeping MG, Meyer JH (2009). Silicon-augmented resistance of plants to herbivorous insects: a review. Annals of Applied Biology 155:171-186.

Reynolds OL, Padula MP, Zeng R, Gurr GM (2016). Silicon: potential to promote direct and indirect effects on plant defense against arthropod pests in agriculture. Frontiers in Plant Science 7:744 doi: 10.3389/fpls.2016.00744.

Santivanez T, Mora Aguilera G, Diaz Pinilla G, Lopez Arrollo JI, Vernal Hurtado P (2013). Citrus. Marco estratégico para la gestión regional del Huanglongbingen América Latina y el Caribe. FAO, Santiago de Chile. Schwachtje J, Baldwin IT (2008). Why does herbivore attack reconfigure primary metabolism?. Plant Physiology 146(3):845-851.

Sétamou M, Flores D, French JV, Hall DG (2008). Dispersion patterns and sampling plans for Diaphorina citri (Hemiptera: Psyllidae) in citrus. Journal of Economy Entomology 101:1478-1487.

Staley JT, Stafford DB, Green ER, Leather SR, Rossiter JT, Poppy GM, Wright DJ (2011). Plant nutrient supply determines competition between phytophagous insects. Proceedings of The Royal Society B 278:718-724.

Tsagkarakis AE, Rogers ME, Spann TA (2012). Applications of plant growth regulators to container-grown citrus trees affect the biology and behavior of the Asian Citrus Psyllid. Journal of The American Society for Horticultural Science 137(1):3-10.

Vieira DL, Barbosa VO, Oliveira de Souza WC, Gonçalves da Silva J, Malaquias JB, Batista JL (2016). Potassium silicate-induced resistance against blackfly in seedlings of Citrus reticulate. Fruits 71(1):49-55.

Wang M,Zheng Q, Shen Q, Guo S (2013). The critical role of potassium in plant stress response. International Journal of Molecular Sciences 14(4):7370-7390.

War AR, Paulraj MG, Ahmad T, Buhroo AA, Hussain B, Ignacimuthu S, Sharma HC (2012). Mechanisms of plant defense against insect herbivores. Plant Signal Behavior 7(10):1306-1320. 